TITLE:

\title{
Instability-based mechanism for body undulations in centipede locomotion
}

$\operatorname{AUTHOR}(S):$

Aoi, Shinya; Egi, Yoshimasa; Tsuchiya, Kazuo

CITATION:

Aoi, Shinya ... [et al]. Instability-based mechanism for body undulations in centipede locomotion. Physical Review E 2013, 87(1): 012717.

ISSUE DATE:

2013-01-22

URL:

http://hdl.handle.net/2433/188009

RIGHT:

(C)2013 American Physical Society 
PHYSICAL REVIEW E 87, 012717 (2013)

\title{
Instability-based mechanism for body undulations in centipede locomotion
}

\author{
Shinya Aoi, ${ }^{1,3}$ Yoshimasa Egi, ${ }^{1}$ and Kazuo Tsuchiya ${ }^{2,3}$ \\ ${ }^{1}$ Department of Aeronautics and Astronautics, Graduate School of Engineering, Kyoto University, \\ Yoshida-honmachi, Sakyo-ku, Kyoto 606-8501, Japan \\ ${ }^{2}$ Department of Energy and Mechanical Engineering, Faculty of Science and Engineering, \\ Doshisha University, 1-3 Tatara, Miyakodani, Kyotanabe, Kyoto 610-0394, Japan \\ ${ }^{3}$ JST, CREST, 5 Sanbancho, Chiyoda-ku, Tokyo 102-0075, Japan
}

(Received 30 May 2011; revised manuscript received 22 November 2012; published 22 January 2013)

\begin{abstract}
Centipedes have many body segments and legs and they generate body undulations during terrestrial locomotion. Centipede locomotion has the characteristic that body undulations are absent at low speeds but appear at faster speeds; furthermore, their amplitude and wavelength increase with increasing speed. There are conflicting reports regarding whether the muscles along the body axis resist or support these body undulations and the underlying mechanisms responsible for the body undulations remain largely unclear. In the present study, we investigated centipede locomotion dynamics using computer simulation with a body-mechanical model and experiment with a centipede-like robot and then conducted dynamic analysis with a simple model to clarify the mechanism. The results reveal that body undulations in these models occur due to an instability caused by a supercritical Hopf bifurcation. We subsequently compared these results with data obtained using actual centipedes. The model and actual centipedes exhibit similar dynamic properties, despite centipedes being complex, nonlinear dynamic systems. Based on our findings, we propose a possible passive mechanism for body undulations in centipedes, similar to a follower force or jackknife instability. We also discuss the roles of the muscles along the body axis in generating body undulations in terms of our physical model.
\end{abstract}

DOI: 10.1103/PhysRevE.87.012717

PACS number(s): 87.19.ru, 87.85.gj

\section{INTRODUCTION}

Centipedes are arthropods that consist of many body segments with one pair of legs per body segment. During terrestrial locomotion, centipedes flex their body segments and move their legs, generating posteriorly propagating traveling waves. Centipede locomotion has the characteristic that body undulations are absent at low speeds, but appear at faster speeds; furthermore, their amplitude and wavelength increase with increasing speed (Fig. 1) [1-3]. By observing centipede locomotion, Manton [2,3] found that rapid stepping movements give rise to these body undulations. According to Manton, such undulations impede centipede motion and are resisted by the muscles along the body axis. Centipedes can dampen body undulations through muscular resistance only at low speeds. This type of motion differs greatly from the axial movements of vertebrates whose locomotion is enhanced by increasing the stride length and the locomotion speed through coordinating the body axis and limb movements [4].

The suggestions of Manton have gained wide acceptance in both the primary scientific literature and textbooks [4-7]. However, Anderson et al. [1] reported that electromyogram measurements of centipedes suggest that muscle activities along the body axis support body undulations rather than resist them. Thus, Anderson et al. and Manton disagree regarding the roles of the muscles and the mechanisms responsible for producing body undulations in centipedes remain largely unclear.

To date, the abilities of animals to generate motion have been investigated by examining the configurations and activities of neural networks. For example, neurophysiological studies of lampreys and cats have greatly contributed to elucidating the locomotor mechanisms of animals [8-13].
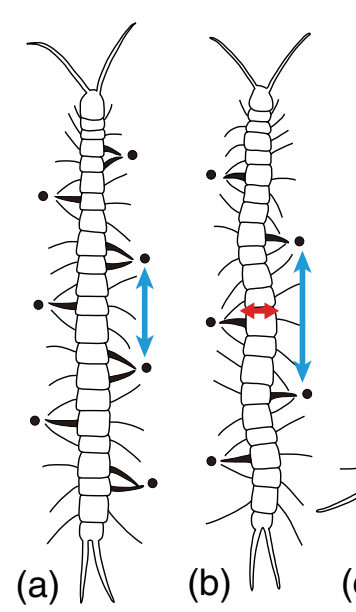

(b)

(c)

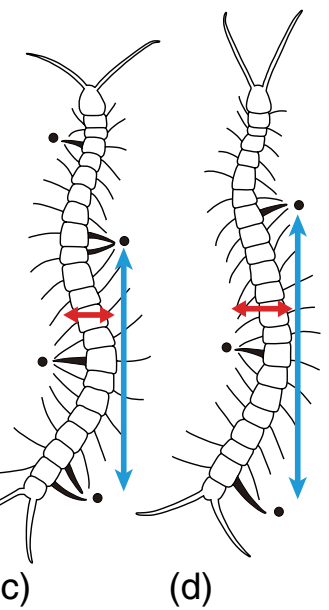

FIG. 1. (Color online) Locomotor behavior of centipede Scolopendra cingulata (modified from Ref. [2]). Locomotion speed increases from (a) to (d). Body undulations are absent at low speeds in (a). Body undulations are present in (b)-(d) and their amplitude and wavelength increase with increasing speed.

Regarding myriapod (e.g., centipedes and millipedes) locomotion, Golubitsky et al. [14,15] proposed a nervous system model to investigate the phase relationship between the leg movements for the gait pattern to produce body undulations, suggesting the presence of important structural characteristics in the central pattern generator network. However, since locomotion is a well-organized motion generated through dynamic interactions among the body, the nervous system, and the environment, it is difficult to fully understand its mechanisms in terms of only the nervous system. In addition to understanding the nervous system, it is crucial to elucidate 
dynamic characteristics inherent in the body. Studies of the nervous and musculoskeletal systems must complement each other to clarify the mechanisms by which animals generate locomotion.

Simple physical models have been used to investigate the roles of body-mechanical systems [16-24]. The stability mechanism is an important factor in locomotion dynamics and bifurcation structures obtained using simple models have provided meaningful biological insights, e.g., a saddle-node bifurcation in the hysteresis in the walk-trot transition of quadrupeds [17]. In a previous study, we performed a simulation that revealed that a multilegged robot system with three or more body segments produces body undulations through a Hopf bifurcation when the joint stiffness is varied [25]. Inspired by this finding, the present study employs a simple body-mechanical model and a robot, which are based on actual centipedes, to investigate the dynamics of centipede locomotion. In particular, we examine whether rapid stepping movements induce body undulations and the mechanism that produces the body undulations. We then compare our results with measured data for actual centipedes. The results obtained reveal similar dynamic characteristics between our model and centipedes, despite centipedes being complex, nonlinear dynamic systems. This implies that a common principle underlies these dynamics and suggests a possible mechanism for the body undulations in centipedes. In addition, from our physical model, we discuss the roles of the muscles on body undulations in terms of the above-mentioned conflicting reports.

\section{DO RAPID STEPPING MOVEMENTS INDUCE BODY UNDULATIONS?}

\section{A. Body-mechanical model and robot}

Our body mechanical model consists of six homogenous modules [Fig. 2(a)]. Each module consists of a single rigid body and one pair of legs. The body and legs are designed to have the simplest mechanical structure with the smallest number of degrees of freedom to obtain the target behavior. Although more modules are preferable for comparison with actual centipedes, we used six modules due to the limitations of the robot experiment described below. Each leg consists of two rigid links that are connected to one another and to the body by pitch joints. The bodies are connected by yaw joints installed with torsional springs (spring constant: $1.8 \mathrm{Nmm} / \mathrm{deg}$ ) to emulate the resistance from the muscular activities of centipedes. Table I lists the physical parameters of each module.

We simplified the role of the nervous system that controls the legs by assuming that the leg tips perform a simple periodic movement relative to the bodies. During locomotion, the legs support the body segments and generate propulsive forces by creating periodic movements relative to the body segments. To generate such leg movements, the length between the root and the leg tip and the orientation must be controlled. We generate leg movement using two pitch joints composed of two parts: half of an elliptical curve that starts from the posterior extreme position (PEP) and ends at the anterior extreme position (AEP) and a straight line from the AEP to the PEP [Fig. 2(a)]. In the straight line section, the leg tips move from the AEP to the PEP
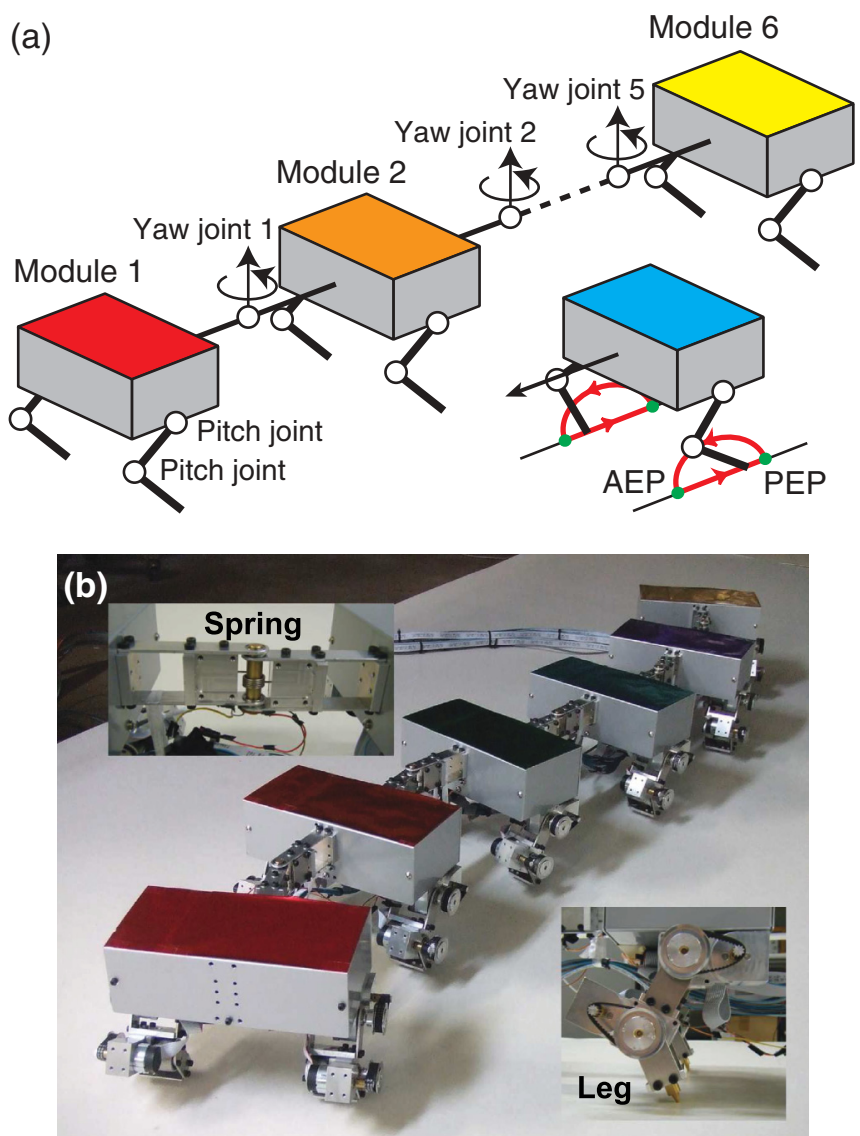

FIG. 2. (Color online) (a) Body-mechanical model and (b) robot. It consists of six homogenous modules that each have one rigid body and one pair of legs. Legs are driven so the leg tips follow a periodic trajectory in the pitch plane including the anterior extreme position (AEP) and the posterior extreme position (PEP) (red line). Bodies are passively connected by yaw joints with installed torsional springs.

in the opposite walking direction at a constant speed parallel to the body. The body is propelled in the walking direction at the constant speed while the leg tips contact the floor. Using $\tau_{\mathrm{el}}$ to denote the duration of the half elliptical curve, $\tau_{\mathrm{st}}$ to denote the duration of the straight line, and $d_{\mathrm{AP}}$ to denote the distance between the AEP and the PEP on each body, the locomotion frequency $\omega$, the stride length $s$, and the locomotion speed $v$ are given by

$$
\omega=\frac{2 \pi}{\tau_{\mathrm{el}}+\tau_{\mathrm{st}}}, \quad s=\frac{\tau_{\mathrm{el}}+\tau_{\mathrm{st}}}{\tau_{\mathrm{st}}} d_{\mathrm{AP}}, \quad v=\frac{d_{\mathrm{AP}}}{\tau_{\mathrm{st}}} .
$$

TABLE I. Physical parameters of each module.

\begin{tabular}{lll}
\hline \hline Link & Parameter & Value \\
\hline Body & Mass (kg) & 0.9 \\
& Length (m) & 0.10 \\
& Width (m) & 0.16 \\
Upper leg & Height (m) & 0.07 \\
& Mass (kg) & 0.3 \\
Lower leg & Length (m) & 0.05 \\
& Mass (kg) & 0.03 \\
& Length (m) & 0.05 \\
\hline \hline
\end{tabular}


Since centipedes control their locomotion speed by adjusting the duration of the propulsive stroke [1-3], we use $d_{\mathrm{AP}}=3 \mathrm{~cm}$ and $\tau_{\mathrm{el}}=0.3 \mathrm{~s}$ and vary the locomotion speed $v$ by changing the duration $\tau_{\text {st }}$ from 0.37 to $0.30 \mathrm{~s}$ to investigate the effect of changing the stepping rate and the locomotion speed on the locomotor behavior. The contralateral legs in each module are manipulated to move in antiphase in a similar manner to that observed in centipedes. The phase difference between the movements of ipsilateral legs on adjacent modules is set to $2 \pi / 3 \mathrm{rad}$.

To simulate the locomotor behavior of our body-mechanical model, we derived the equation of motion using Lagrangian equations as in Ref. [17]. Contact between the leg tips and the floor was modeled by vertical viscoelastic elements and horizontal viscous elements. Joint friction was modeled by linear damping. We numerically solved the equation of motion using the fourth-order Runge-Kutta method with a step size of $1 \mathrm{~ms}$.

In addition to performing a computer simulation based on this body-mechanical model, we designed a centipede-like robot based on this model to demonstrate its real-world dynamic characteristics [Fig. 2(b)]. We did this since robots are very effective and powerful tools for testing hypotheses regarding real-world phenomena [20,26-35]. In the robot experiments, the robot walked on a flat surface $\left(180 \times 990 \mathrm{~cm}^{2}\right)$. Variations in the elevation of this surface are smaller than $3 \mathrm{~mm}$, which have little, if any, effect on the walking behavior of the robot. The robot has an external power supply and is controlled by an external host computer (Intel Pentium $42.8 \mathrm{GHz}$, RT-Linux) that calculates the desired motions of the leg joints and sends command signals in 1-ms intervals. The cables running from the robot to the external power supply and to the host computer were raised during experiments to prevent them from affecting the walking behavior.

\section{B. Appearance of body undulations}

We performed simulations and robot experiments for a walk in a straight line on a flat floor, where we set all the yaw joint angles to zero as the initial condition. Since torsional springs are installed on the yaw joints and the leg tips move parallel to the body segments with an identical stepping rate for all the legs, both the model and the robot are expected to walk in a straight line while keeping their body segments parallel to each other. They actually performed a walk in a straight line and body undulations were absent at low speeds, similarly to the locomotor behavior of the centipedes at low speeds [Fig. 1(a)]. However, when the stepping rate and the locomotion speed were increased and after the initial transient of about $20 \mathrm{~s}$, lateral undulations among the body segments appeared above a critical speed despite the absence of periodic control signals in the yaw joints [Figs. 3(a) and 3(b)].

We next investigated the effects of the stepping rate and the locomotion speed on the body undulations. Although we did not find a significant dependence of the undulation speed (body length/period of body undulations) on the locomotion speed, the amplitude and wavelength of the body undulations exhibited a clear dependence on the locomotion speed, similarly to case for actual centipedes in Fig. 1. To investigate them, we examined the amplitude and the phase lag of the undulatory movements of the yaw joints at various
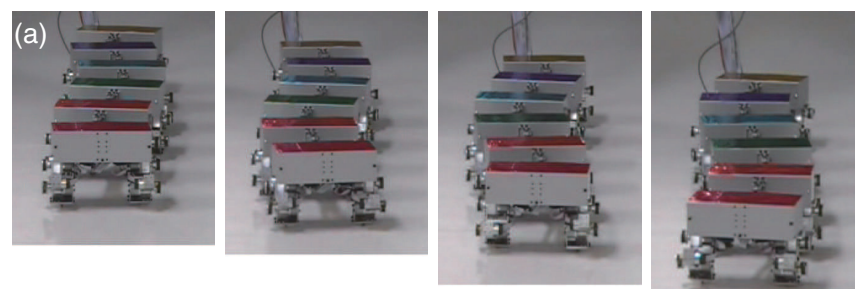

(b)
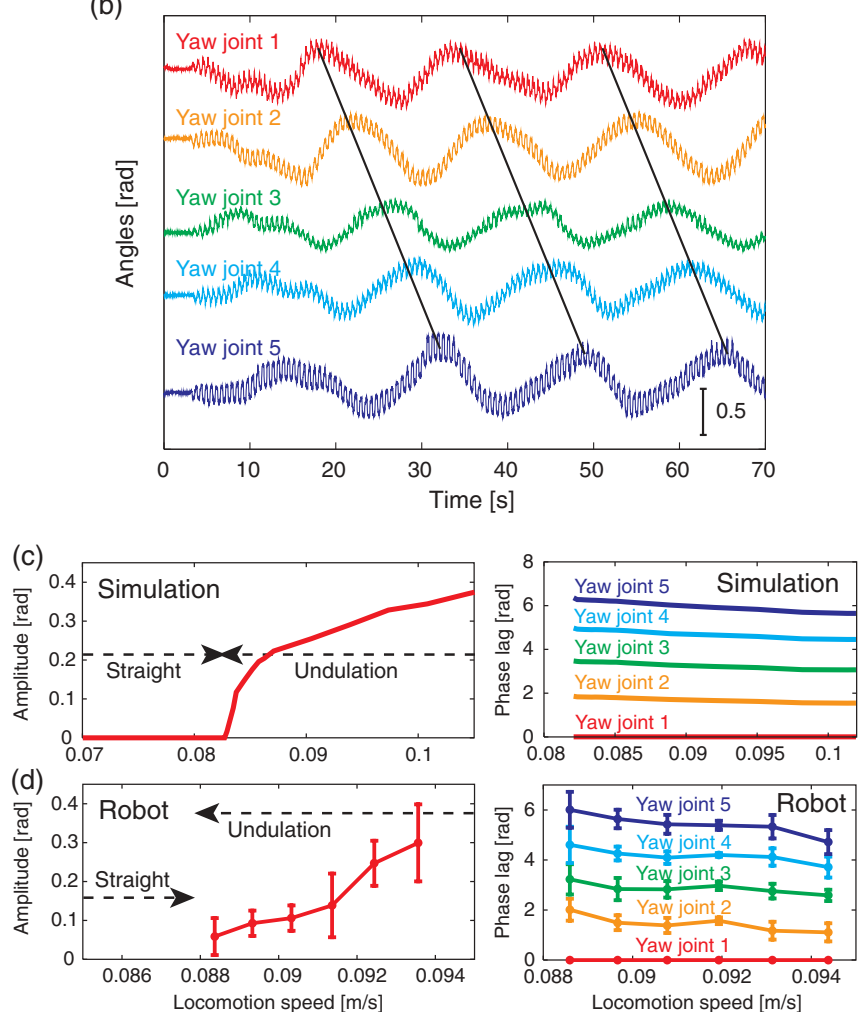

FIG. 3. (Color online) Appearance of body undulations. (a) Snapshot (see supplementary movie [36]) and (b) yaw angle profile during robot experiment (stepping period: $0.6 \mathrm{~s}$ and period of body undulations: $16 \mathrm{~s}$ ). [(c) and (d)] The changes in the amplitude and phase lag of body undulations in the simulation and robot, respectively. The data points and error bars in (d) respectively correspond to the means and standard deviations of the results of five experiments.

locomotion speeds. Figures 3(c) and 3(d) show the results of the simulation and robot experiments [36]. The amplitude shown is that of yaw joint 3. The phase lag is calculated by taking the Fourier transform and it is represented by the lag from yaw joint 1. The data points and error bars for the robot experiments correspond to the means and standard deviations of five experiments, respectively. The simulation and robot experiments give similar results. The amplitude of the body undulations increases and the phase lag decreases with increasing locomotion speed.

\section{WHAT MECHANISM PRODUCES THE BODY UNDULATIONS?}

\section{A. Simple model}

To clearly determine the dynamic mechanism of our model and robot, we created a simple model by constructing a 


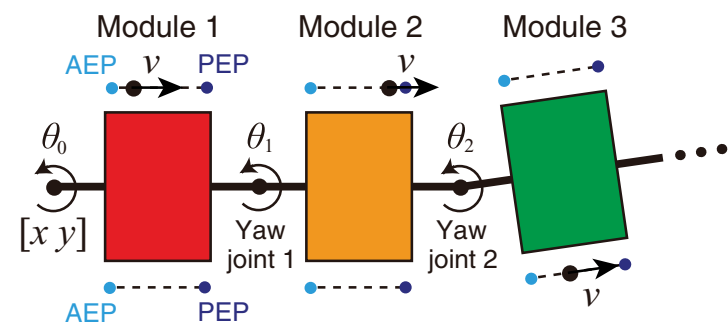

FIG. 4. (Color online) Simple model in two-dimensional space. $x$ and $y$ are the coordinates of the first module, $\theta_{0}$ is the yaw angle of the first module relative to the floor, and $\theta_{i}(i=1, \ldots, 5)$ are the yaw joint angles. Black dots beside the bodies indicate the positions of the leg tips during the stance phase that move parallel to bodies at constant speed $v$ relative to the bodies from the anterior extreme position (AEP) to the posterior extreme position (PEP). This model is subject to reaction forces from the floor only when the legs are in the stance phase.

low-dimensional model from the original high-dimensional mechanical model to extract the fundamentals of locomotion dynamics. Since our goal is to investigate the dynamic properties in order to generate undulatory body movements, we made the following assumptions: (1) the dynamic roles of the legs primarily involve reaction forces produced from contact with the floor and the inertial forces of the legs have only a small influence and (2) since several legs contact the floor to support the bodies and the leg movements do not cause the up-and-down, roll, and pitch motions of the bodies, these motions are sufficiently small relative to the other body motions (left-and-right, back-and-forth, and yaw motions).

From these assumptions, the dynamics of our model can be accounted for by employing a simple model defined in a twodimensional space (Fig. 4), where $x$ and $y$ are the coordinates of the first module, $\theta_{0}$ is the yaw angle of the first module relative to the floor, $\theta_{i}(i=1, \ldots, 5)$ is the yaw joint angle, and the black dots besides the bodies indicate the positions of the leg tips during the stance phase that move parallel to the bodies at constant speed $v$. Only when the leg tips are in the stance phase is the model subject to reaction forces from the floor.

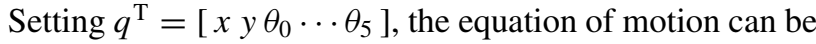
derived using the Lagrangian equation

$$
K(q) \ddot{q}+h(q, \dot{q})=u(q, \dot{q})+\lambda(q, \dot{q}, t ; v),
$$

where $K(q)$ is the inertia matrix, $h(q, \dot{q})$ is the nonlinear term, $u(q, \dot{q})$ is the torque term due to the torsional springs, and $\lambda(q, \dot{q}, t ; v)$ is the reaction force term. Since the leg tips move periodically relative to the body and the speed of the leg tips relative to the body during the stance phase is $v$, the reaction force $\lambda$ becomes a function of time $t$ with parameter $v$. A more specific description of this equation is presented in Appendix A.

Using this simple model, we investigated the mechanisms for producing body undulations from a stability analysis of walking in a straight line, in which our model walks at constant speed $v$ and the bodies remain parallel with each other and do not undulate laterally. To describe the configuration of this model, we used $z^{\mathrm{T}}=\left[q^{\mathrm{T}} \dot{q}^{\mathrm{T}}\right]$. When the model walks in a straight line, we can write $\hat{z}^{\mathrm{T}}=\left[v t+x_{0} y_{0} 0 \cdots 0 v 00 \cdots 0\right]$ $(0 \leqslant t \leqslant \tau)$ for one stepping cycle, where $x_{0}$ and $y_{0}$ are, respectively, the values of $x$ and $y$ at $t=0$ and $\tau$ is the stepping period. Linearization of the equation of motion (2) around $\hat{z}$ yields

$$
\delta \dot{z}=A(t ; v) \delta z,
$$

where $\delta z=z-\hat{z}$ and $A(t ; v)=A(t+\tau ; v)$ since the movements of the leg tips are periodic with period $\tau$. Further details regarding this linearization are given in Appendix A.

The fundamental solution matrix of a linear differential equation with periodic coefficients, such as Eq. (3), can be written in the form

$$
X(t)=Z(t) e^{\Lambda t},
$$

where $X(t)$ is the fundamental solution matrix and $Z(t)=$ $Z(t+\tau)$ [37]. We can use identity matrices for $X(0)$ and $Z(0)$. Integrating the linear differential equation from $t=0$ to $t=\tau$ gives

$$
X(\tau)=Z(\tau) e^{\Lambda \tau}=Z(0) e^{\Lambda \tau}=e^{\Lambda \tau} .
$$

The behavior of the solution is determined by the eigenvalues of the constant matrix $e^{\Lambda \tau}$ (Floquet multipliers). When all the Floquet multipliers lie inside the unit circle on the complex plane or all the real parts of the Floquet exponents (eigenvalues of constant matrix $\Lambda$ ) are negative, the solution is asymptotically stable.

\section{B. Floquet analysis with simple model}

We calculated the Floquet exponents of the linearized equation (3) for each $v$ to investigate the stability of a straight walk with respect to the locomotion speed. Figure 5(a) shows the result. Since the state $\delta z$ in (3) is of dimension 16 , there are 16 lines representing the different Floquet exponents. The region enclosed by the dashed lines is enlarged to show the region near the origin, where the arrows indicate the direction of the changes in the exponents with increasing locomotion speed $v$. Except for zero exponents, all the exponents lie in the left half plane at low speeds. However, with increasing locomotion speed $v$, one pair of exponents crosses the imaginary axis and enters the right half plane. This indicates that a Hopf bifurcation occurs above a critical speed and that the dynamic system becomes unstable. The circles indicate all the exponents when the Hopf bifurcation occurs. The red circles imply the Floquet exponents become unstable on crossing the imaginary axis. We subsequently calculated the phase lags of the yaw joint elements in the destabilizing eigenvector relative to the element of yaw joint 1 for the locomotion speed [Fig. 5(b)] to find the dynamic characteristics after the system is destabilized. The phase lags decrease with increasing speed, which is similar to the results of the simulation and robot experiments [Figs. 3(c) and 3(d)].

This result suggests that when the locomotion speed exceeds the critical speed, the model can no longer continue to walk in a straight line with parallel bodies and the walking behavior changes completely. This also suggests that this destabilization causes the state variables to increase exponentially with time in accordance with the destabilizing eigenvector and that this generates undulatory motion. Comparison of the phase lags of the yaw joint elements between the destabilizing eigenvector and the undulatory behaviors 
(a)

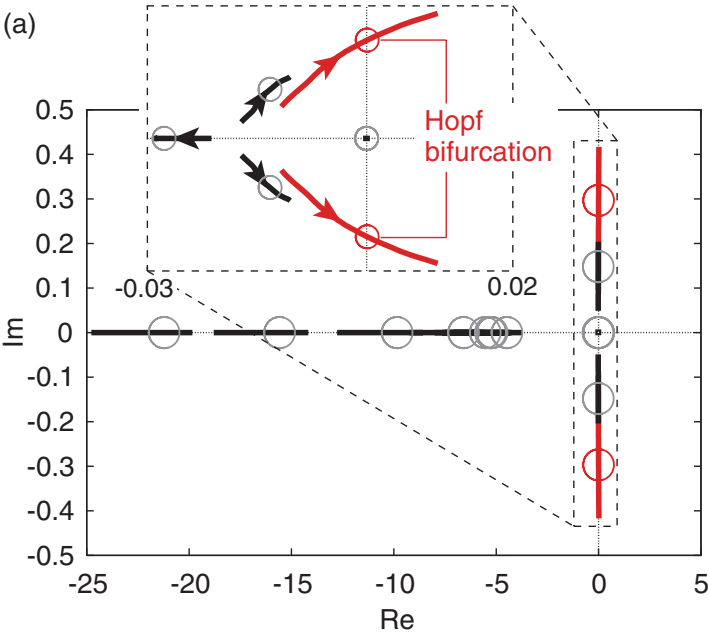

(b)

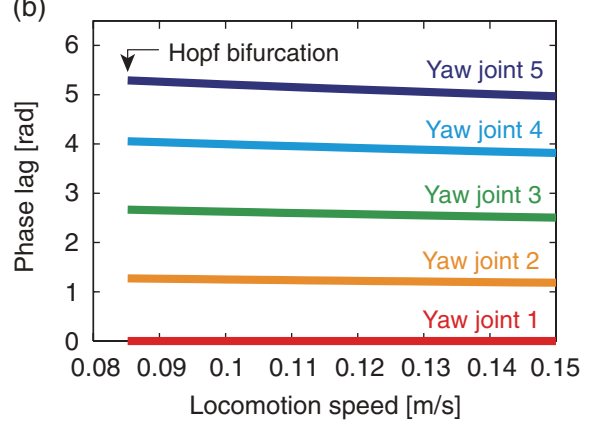

FIG. 5. (Color online) Floquet analysis based on the simple model. (a) Floquet exponents for increasing speed $v$. The region enclosed by dashed lines is enlarged to show the region near the origin. The circles indicate the exponents when Hopf bifurcation occurs. (b) Phase lags of yaw joint elements of the destabilizing eigenvector relative to yaw joint 1 .

of the simulation and robot experiments reveal similarities [Figs. 3(c) and 3(d) and 5(b)], suggesting that the nonlinearity in the dynamics limits the exponential growth and helps complete body undulations. These results indicate that this destabilization gives rise to the body undulations in our model and robot. As the simulation results in Fig. 3(c) show, the amplitude increases proportionally with the square root of the speed, which is a characteristic of the supercritical Hopf bifurcation [38]. These results imply that body undulations do not necessarily require intentional coordinative inputs to the yaw joints; rather they can be induced in passive structures when the dynamics contain such an instability.

\section{Further investigation using nondimensional parameters}

We further analyze the linearized equation (3) using nondimensional parameters to clarify the mechanism to produce body undulations. We introduced a reference time $\xi$ (to be specified later) and the linearized equation (3) can be written as

$$
\delta z^{* \prime}=A\left(\phi^{*} ; w^{*}, j^{*}, k^{*}, d^{*}, \sigma^{*}, \alpha^{*}, v^{*}\right) \delta z^{*},
$$

where $\delta z^{* \mathrm{~T}}=\left[\delta q^{* \prime \mathrm{T}} \delta q^{* \mathrm{~T}}\right], \delta q^{*}=\left[\delta x / l \delta y / l \delta \theta_{0} \cdots \delta \theta_{5}\right]^{\mathrm{T}}$, $\phi^{*}$ is the nondimensional time divided by $\xi\left(\phi^{*}=t / \xi\right)$, and ()$^{\prime}$ indicates the derivative with respect to $\phi^{*}$. This
TABLE II. Stability analysis parameters.

\begin{tabular}{llc}
\hline \hline Symbol & \multicolumn{1}{c}{ Parameter } & Value \\
\hline$m$ & Mass $(\mathrm{kg})$ & 1.6 \\
$j$ & Inertia $\left(\mathrm{kg} \mathrm{m}^{2}\right)$ & 0.009 \\
$l$ & Length $(\mathrm{m})$ & 0.25 \\
$w$ & Width $(\mathrm{m})$ & 0.16 \\
$k$ & Spring coefficient $(\mathrm{Nm})$ & 0.1 \\
$d$ & Damping coefficient $(\mathrm{Nm} \mathrm{s})$ & 3.0 \\
$\sigma$ & Friction coefficient $(\mathrm{Ns} / \mathrm{m})$ & 100 \\
\hline \hline
\end{tabular}

equation is governed by seven nondimensional parameters: $w^{*}=w / l, j^{*}=j /\left(m l^{2}\right), k^{*}=k \xi^{2} /\left(m l^{2}\right), d^{*}=d \xi /\left(m l^{2}\right)$, $\sigma^{*}=\sigma \xi / m, \alpha^{*}=\xi / \tau$, and $v^{*}=v \xi / l$, where $m, j, l$, and $w$ are, respectively, the mass, inertia, length, and width of each module, $k$ and $d$ are, respectively, the spring and damping coefficients in the yaw joints, and $\sigma$ is the friction coefficient of the floor. Table II shows the parameters used for the stability analysis. Further details regarding this analysis using nondimensional parameters are provided in Appendix B.

We investigated the effect of nondimensional parameters on a Hopf bifurcation based on (6). In particular, we examined the effects of parameters $w^{*}, j^{*}, k^{*}, d^{*}, \alpha^{*}$, and $\sigma^{*}$ on the critical speed of $v^{*}$ for producing a Hopf bifurcation. Figure 6(a) shows the result when one nondimensional parameter was varied while the other nondimensional parameters were kept constant. We used the period of the destabilized Floquet exponents at the bifurcation point as the reference time $\xi$ since it corresponds to the period of the body undulations. This figure shows that the spring stiffness $k^{*}$ and the floor friction $\sigma^{*}$ greatly increase and decrease the critical speed, respectively. Figure 6(b) shows the effects of the spring stiffness $k^{*}$ and the floor friction $\sigma^{*}$ on the Floquet exponents. It shows that the spring stiffness shifts the exponents to the left half plane, whereas the floor friction shifts the exponents to the right half plane. These results suggest that the torsional spring is a stabilizing factor in the internal structure for a walk in a straight line, while the floor friction is a destabilizing factor due to external forces driven by the movement of the leg tips parallel to the bodies. The Hopf bifurcation is mainly determined by the relationship between these two factors. Such an instability resembles instabilities in other dynamic systems, such as the flutter instability in a flexible beam subjected to a follower force at the end (e.g., a rocket) [39-42] and the jackknife instability in a car-trailer system in which the car pulls one or more trailers and experiences external forces through the wheels [43-45].

In addition, we investigated the effect of the number of modules on the critical speed. Figure 6(c) shows the result. Although the number of modules used is less than the number of segments in centipedes, this result shows that the number of modules has a small effect on the critical speed.

\section{RELATIONSHIP WITH CENTIPEDES}

\section{A. Comparison of body undulations with centipedes}

In the simulation and robot experiments, body undulations were absent at low speeds but appeared above a critical speed (Fig. 3), similarly to centipedes [2,3]. To further investigate 

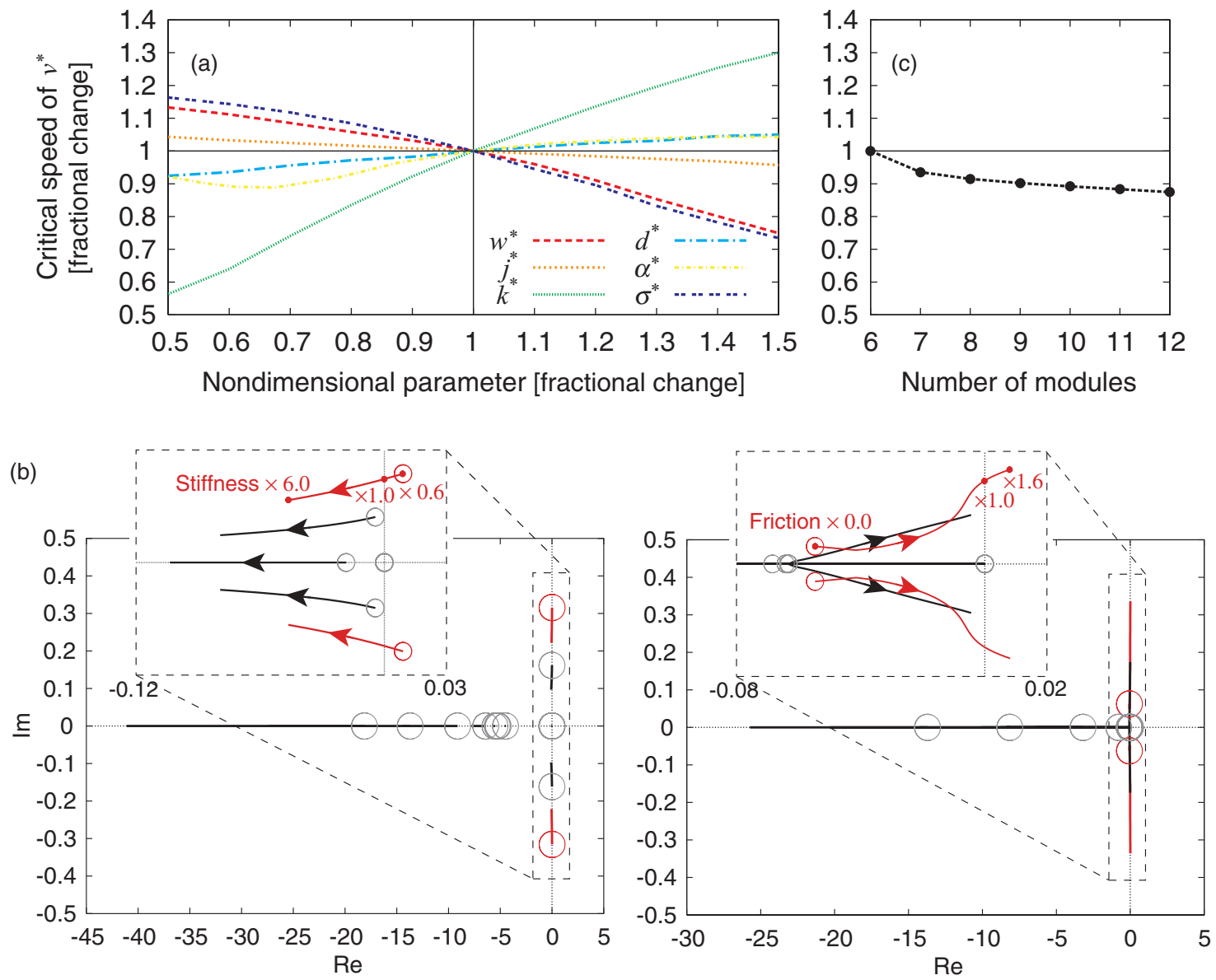

FIG. 6. (Color online) Effect of nondimensional parameters on a Hopf bifurcation. (a) Effects on the critical speed. (b) Effects of spring stiffness $k^{*}$ (left) and floor friction $\sigma^{*}$ (right) on the Floquet exponents. The circles indicate all Floquet exponents for a spring stiffness of $60 \%$ (left) and zero floor friction (right). (c) Effect of number of modules on critical speed.

the biological relevance, we compared the simulation and robot experiments results with data obtained from actual centipedes. Figures 7(a) and 7(b) show the amplitude and the phase lag of body undulations for the simulation and robot experiments, respectively, recalculated from Figs. 3(c) and 3(d) using the nondimensional locomotion speed. Figure 7(c) shows the mean values of the amplitude and the phase lag for three different speeds measured by Anderson et al. [1]. The amplitude shown is that of the lateral flexion between adjacent body segments. The phase lag is calculated from the wavelength of body undulations; it represents the lag between the foremost and rearmost body segments divided by the number of body segments. The data for the three systems are approximately the same and show a similar trend in that the amplitude increases and the phase lag decreases (wavelength increases) with increasing locomotion speed.

\section{B. Effects of spring constants of torsional springs at yaw joints}

Anderson et al. [1] reported that the muscles along the body axis support the body undulations during centipede locomotion, whereas Manton [2,3] suggested that the muscles resist the undulations. In the present study, we used torsional springs installed at the yaw joints to emulate the resistance from the muscular activities of centipedes suggested by
Manton. To investigate the effects of the torsional springs on the locomotion dynamics, we changed the spring constants in the simulation experiments. We reduced the spring constants at a low speed during locomotion without varying the parameters for the locomotion speed and we examined how this change influences the body undulations. In this experiment, before the spring constants reach zero, the model performs locomotion with body undulations for a certain duration, but the undulations are not maintained and the locomotion collapses when the amplitude of body undulations becomes too large to walk forward [36].

To more clearly elucidate these effects, we used various values for the spring constants and examined if the body undulations are maintained long enough for each value. The results are shown in Fig. 8, which plots the amplitude of yaw joint 3 when the body undulations are maintained. Body undulations are absent for large spring constants but appear in a certain range of the spring constant. Comparison with Fig. 7(b) reveals that the contribution of the increase (decrease) in the spring constant to the production of body undulations is similar to that of the decrease (increase) in the locomotion speed. Locomotion collapses below a certain value of the spring constant. Our results indicate that our model requires dynamic factors to resist body undulations to generate locomotion, which is similar to the suggestion by Manton. 
(a)

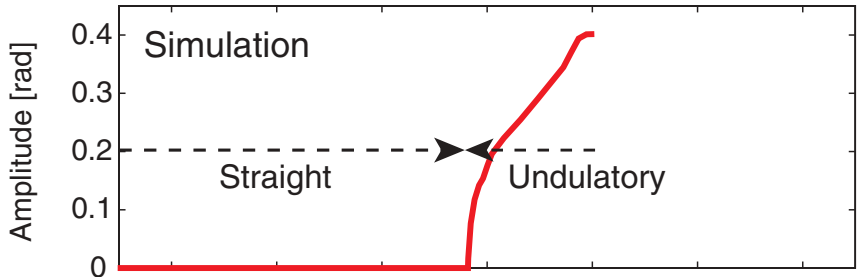

(b)

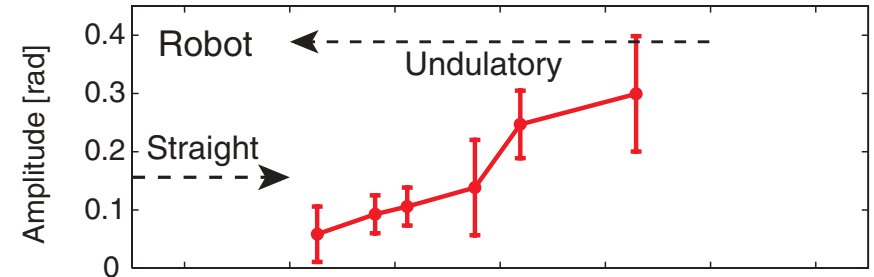

(c)

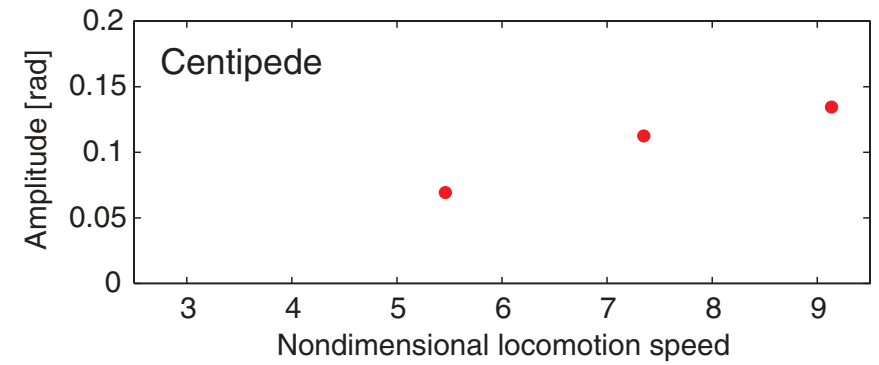

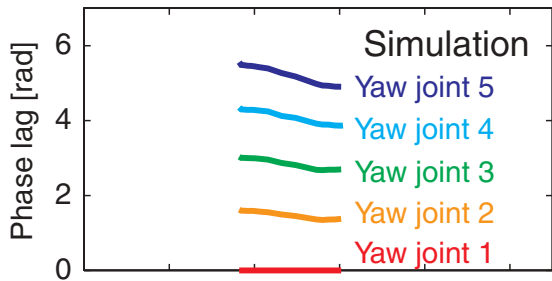
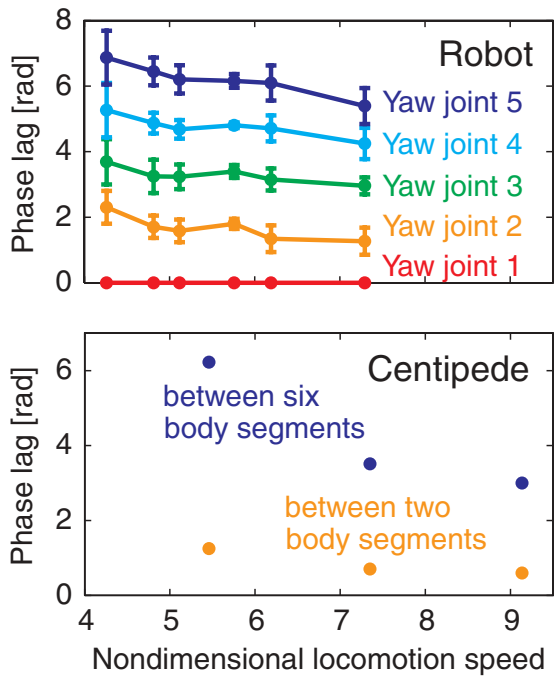

FIG. 7. (Color online) Comparison of the amplitude and phase lag of body undulations among (a) simulation, (b) robot, and (c) actual centipedes using nondimensional locomotion speed. Panels (a) and (b) are recalculated from Figs. 3(c) and 3(d). (c) The mean values measured by Anderson et al. [1].

\section{Effects of active inputs in the yaw joints}

Anderson et al. [1] demonstrated that the muscles along the body axis promote body undulations during centipede locomotion, where the muscle activity alternates between the left and right sides for each body segment. We also investigated the effect of this active muscle activity on body undulations by applying periodic torques to the yaw joints in the simulation experiments. Specifically, we used a spring constant of $2.5 \mathrm{Nmm} / \mathrm{deg}$ for the torsional springs of the yaw joints, which did not induce body undulations in Fig. 8, and we input periodic torques to the yaw joints. Thus, we used both passive and active contributions to the yaw joints. We used a

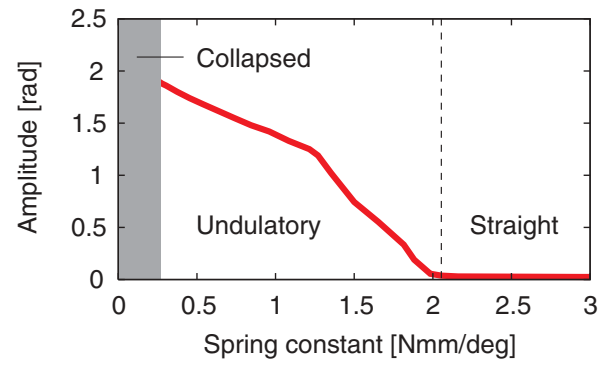

FIG. 8. (Color online) Amplitude of undulatory movements in yaw joint 3 for the spring constants obtained in simulation experiments. The amplitude increases with decreasing spring constant. When the spring constant is sufficiently large, body undulations are absent. Locomotion collapses when the spring constant is below a certain value. Body undulations appear over a certain range of the spring constant. sine function for the periodic inputs. We determined the period of the periodic inputs and the relative amplitude and relative phase of the periodic inputs between the yaw joints based on the undulatory movements of the yaw joints in the above result using a spring constant of $1.8 \mathrm{Nmm} / \mathrm{deg}$. We used various values for the amplitude of this active input and investigated if body undulations appear. Figure 9 shows the result of the amplitude of the undulatory movements in yaw joint 3 . As shown in this figure, body undulations appeared in response to the active input, similar to the suggestion by Anderson et al.

\section{Conflicting views regarding the role of the muscles}

In this study, we used two models to investigate the role of the muscles along the body axis of centipedes. One model

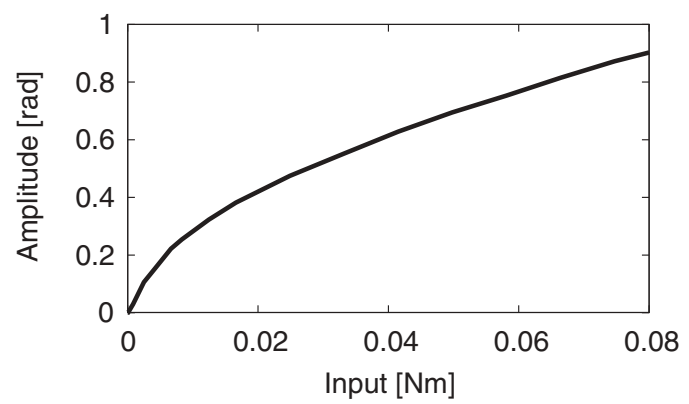

FIG. 9. Amplitude of undulatory movements in yaw joint 3 for the amplitude of the active input obtained in simulation experiments. The amplitude of the undulatory movements increases with increasing amplitude of the active input. 
uses torsional springs in the yaw joints to model the resistance from the muscular activities of centipedes, as suggested by Manton [2,3]. In this case, we found that body undulations occur due to an instability caused by a supercritical Hopf bifurcation. The other model employs periodic torque inputs to the yaw joints to model the active contribution of the muscles, as suggested by Anderson et al. [1]. In this case, we also used torsional springs since dynamic analysis revealed that our model requires dynamic factors to resist body undulations to continue locomotion. Therefore, this case assumes that the passive elements in the muscular and body-mechanical systems contribute to the resistance to the movements of the yaw joints. Our results showed that this active input also produced similar body undulations to those generated through the Hopf bifurcation. This means that our physical model can explain the dynamic contributions of the muscles to the generation of undulatory body movements, as suggested by Manton and Anderson et al. using these different models. However, these models generate body undulations by different mechanisms.

\section{LIMITATIONS OF OUR APPROACH AND FUTURE WORK}

We developed a simple body-mechanical model inspired by centipedes. However, there are differences between the model and real centipedes. Regarding morphology, our model has only six bodies and 12 legs. The legs extend downward and the physical properties are nearly identical among the bodies and the legs. In contrast, centipedes have 15 or more body segments [46], their legs project laterally, and the physical properties, such as mass and size, differ among the body segments and legs. For the locomotor behavior, the lateral undulations of the body segments and the stepping movements during centipede locomotion are synchronized in a way that the propulsive legs are located on the concave side of each bent region [1-3]. In contrast, the period of the body undulations during robot locomotion is longer than that of the stepping movements [Fig. 3(b)]. Furthermore, while we used a constant phase difference between successive legs regardless of the locomotion speed, the phase difference during centipede locomotion varies with speed due to the synchronization of the body undulations and the stepping movements. Our models do not explain the mechanisms for this synchronization. In addition, the period of body undulations in centipedes has been measured in to be $0.2-0.6 \mathrm{~s}$ [1], which differs greatly from that of our robot (about $16 \mathrm{~s}$ ). These differences are due the limitations of the robot employed in this study. However, the results for the simulation, the robot, and actual centipedes approximately agree with each other in terms of the nondimensional locomotion speed (Fig. 7) and the nondimensional analysis reveals that the difference in the period of body undulations and stepping movements $\alpha^{*}$ and the number of body segments have little effect [Figs. 6(a) and 6(c)]. From the nondimensional spring stiffness $k^{*}$, the spring constant of actual centipedes is estimated to be $3.1 \mathrm{mNmm} / \mathrm{deg}$, which produces a torque of $24 \mathrm{mNmm}$ from the amplitude of the highest speed in Fig. 7(c). This torque can be produced by a muscle tension of $12 \mathrm{mN}$ when the corresponding muscles are attached at a point $2 \mathrm{~mm}$ from the yaw axis, which is not an unrealistic value $[47,48]$.
The limitations in terms of the synchronization of body undulations and leg movements of our model partially reflect the fact that our model does not incorporate an elaborate nervous system model. The nervous system model by Golubitsky et al. $[14,15]$ explains the phase relationship between the leg movements and body undulations, although it does not explain the mechanical mechanisms that produce the body undulations. A nervous system model alone or a mechanical system model alone cannot fully explain the mechanisms since neuromechanical interactions are crucial in animal locomotion. We intend to improve our model by integrating body mechanical and nervous system models in future studies.

In our stability analysis, the linearized equation (A6) is a second-order system whose proportional and derivative coefficients depend on the summation of the spring and damping coefficients in the yaw joints and periodic coefficients induced by the reaction force from the floor. The stability of a flexible beam subjected to a periodic follower force has been described by coupled Mathieu equations and parametric resonance [40]. The stability mechanism of our models may be related to that of the follower force problem and it would be better to use a characteristic elastic time than the period of body undulations for the reference time $\xi$. However, one aim of this study is to compare the physical characteristics of our models with those of actual centipedes. The spring coefficient of centipedes is currently unknown, which prevents us from determining the characteristic elastic time of centipedes and from comparing our results with those obtained from centipedes. We instead consistently used the period of body undulations as the reference time $\xi$ to conduct our analysis and compare our results with those for centipedes. This allowed the spring coefficient in centipedes to be estimated by nondimensional analysis, as explained above. We intend to use the characteristic elastic time to investigate the relationship between our results and parametric resonance in the follower force problem in a future study.

In conclusion, although the above-mentioned differences and limitations exist, dynamic analysis revealed many similarities between our model and actual centipedes, including the appearance of body undulations during rapid stepping movements and similar trends in the variation in the body undulation amplitude and wavelength as a function of speed (Fig. 7). Despite centipedes being complex, nonlinear dynamic systems, these similarities suggest that our findings provide a possible mechanism for the production of body undulations in centipedes. In addition, simulations performed using two different models for the physical contribution of the muscles along the body axis will provide new insights into the underlying mechanism. More thorough investigations are required to clarify the underlying mechanism in centipede locomotion. However, the possible mechanism and additional insight into the conflicting views regarding dynamics will provide new insights into biological science.

\section{ACKNOWLEDGMENTS}

We thank Avis H. Cohen and Eric D. Tytell for helpful comments regarding an earlier version of the manuscript and thank the anonymous reviewers for valuable comments on this manuscript. We also thank Tsuyoshi Yamashita and 
Takahiro Kondo for help with the experiments. This paper is supported in part by a Grant-in-Aid for Scientific Research (B) No. 23360111, a Grant-in-Aid for Challenging Exploratory Research No. 23656185, and a Grant-in-Aid for Creative Scientific Research No. 19GS0208 from the Ministry of Education, Culture, Sports, Science, and Technology of Japan.

\section{APPENDIX A: PREPARATIONS FOR FLOQUET ANALYSIS WITH A SIMPLE MODEL}

Here, we present the analytical preparations to performing Floquet analysis with a simple model (Fig. 4) by modifying the analysis in our previous study [25] to investigate the influence of the locomotion speed on the locomotion dynamics of our body-mechanical model and robot. The details of the equation of motion of the simple model (2) are as follows. The inertia matrix $K(q)$ is given by

$$
K(q)=H^{\mathrm{T}}\left\{L(q)^{\mathrm{T}} M L(q)+J\right\} H
$$

where

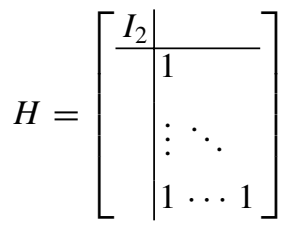

$$
\begin{aligned}
& L(q)=L_{1}(q)+L_{2}
\end{aligned}
$$

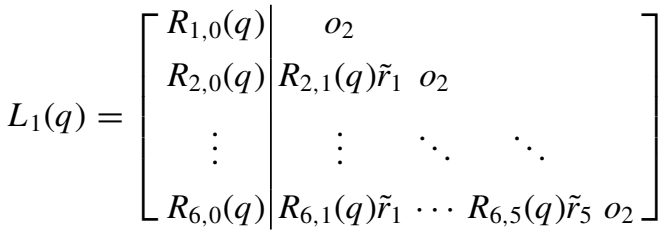

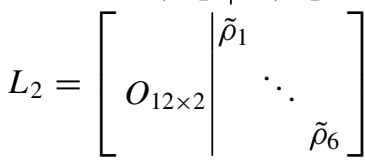

$$
\begin{aligned}
& M=\operatorname{diag}\left[\begin{array}{lllllll}
m_{1} & m_{1} & m_{2} & m_{2} & \cdots & m_{6} & m_{6}
\end{array}\right] \\
& J=\operatorname{diag}\left[\begin{array}{llllll}
0 & 0 & j_{1} & j_{2} & \cdots & j_{6}
\end{array}\right]
\end{aligned}
$$

$m_{i}$ and $j_{i}(i=1, \ldots, 6)$ are the mass and inertia of module $i, o_{2}$ is a zero vector, $I_{n}$ is a $n \times n$ identity matrix, $O_{n \times m}$ is a $n \times m$ zero matrix, $r_{i}(i=1, \ldots, 5)$ is the distance vector from yaw joint $i-1$ to yaw joint $i$ (we assume the position of module 1 indicated by $\left[\begin{array}{ll}x & y\end{array}\right]$ in Fig. 4 as yaw joint 0$), \rho_{i}$ $(i=1, \ldots, 6)$ is the distance vector from yaw joint $i-1$ to the center of mass of module $i$, and $R_{i, j}(q)(i, j=0, \ldots, 6$, $i>j$ ) is the rotation matrix given by

$$
\begin{gathered}
R_{i, j}(q)=\left[\begin{array}{cc}
\cos \theta_{i, j}(q) & \sin \theta_{i, j}(q) \\
-\sin \theta_{i, j}(q) & \cos \theta_{i, j}(q)
\end{array}\right], \\
i, j=0, \ldots, 6, i>j
\end{gathered}
$$

where

$$
\theta_{i, j}(q)=\sum_{k=j}^{i-1} \theta_{k}
$$

and for vector $b=\left[b_{1} b_{2}\right]^{\mathrm{T}}, \tilde{b}$ is expressed as $\tilde{b}=\left[-b_{2} b_{1}\right]^{\mathrm{T}}$. The nonlinear term $h(q, \dot{q})$ is expressed by

$$
h(q, \dot{q})=\dot{K}(q, \dot{q}) \dot{q}+V(q, \dot{q}) p(q, \dot{q}),
$$

where

$$
\begin{aligned}
& V(q, \dot{q})=\left[\begin{array}{ccc}
\frac{O_{2 \times 12}}{\tilde{v}_{1}^{\mathrm{T}}(q, \dot{q})} & & \\
& \ddots & \\
& & \tilde{v}_{6}^{\mathrm{T}}(q, \dot{q})
\end{array}\right] \\
& {\left[v_{1}^{\mathrm{T}}(q, \dot{q}) \ldots v_{6}^{\mathrm{T}}(q, \dot{q})\right]^{\mathrm{T}}=L_{1}(q) H \dot{q}} \\
& p(q, \dot{q})=B(q)^{\mathrm{T}} M L(q) H \dot{q} \\
& B(q)=\left[\begin{array}{ccc}
I_{2} & & \\
R_{2,1}(q) & I_{2} & \\
\vdots & \ddots & \ddots \\
R_{6,1}(q) & \cdots & R_{6,5}(q) I_{2}
\end{array}\right] .
\end{aligned}
$$

The torque term due to the torsional springs $u(q, \dot{q})$ becomes equivalent to

$$
u(q, \dot{q})=-P q-D \dot{q},
$$

where

$$
\begin{aligned}
& P=\operatorname{diag}\left[\begin{array}{llllll}
0 & 0 & 0 & k & \cdots & k
\end{array}\right] \\
& D=\operatorname{diag}\left[\begin{array}{llllll}
0 & 0 & 0 & d & \cdots & \cdots
\end{array}\right]
\end{aligned}
$$

and $k$ and $d$ are the spring and damping coefficients in the yaw joints. Since the horizontal reaction force between the leg tips and the floor is modeled by viscous elements, the reaction force term $\lambda(q, \dot{q}, t ; v)$ becomes

$$
\begin{aligned}
& \lambda(q, \dot{q}, t ; v) \\
& \quad=-H^{\mathrm{T}} L_{\mathrm{tip}}(q, t ; v)^{\mathrm{T}} \Lambda_{\mathrm{tip}}(t)\left\{L_{\mathrm{tip}}(q, t ; v) H \dot{q}+v_{\text {tip }}(t ; v)\right\},
\end{aligned}
$$

where

$$
\begin{aligned}
& L_{\text {tip }}(q, t ; v)= \\
& {\left[\begin{array}{c|ccc}
R_{1,0}(q) & \tilde{l}_{1}^{1}(t ; v) & & \\
R_{1,0}(q) & \tilde{l}_{1}^{2}(t ; v) & & \\
R_{2,0}(q) & R_{2,1}(q) \tilde{r}_{1} & \tilde{l}_{2}^{1}(t ; v) & \\
R_{2,0}(q) & R_{2,1}(q) \tilde{r}_{1} & \tilde{l}_{2}^{2}(t ; v) & \\
\vdots & \vdots & \ddots & \ddots \\
R_{6,0}(q) & R_{6,1}(q) \tilde{r}_{1} & \cdots & R_{6,5}(q) \tilde{r}_{5} \tilde{l}_{6}^{1}(t ; v) \\
R_{6,0}(q) & R_{6,1}(q) \tilde{r}_{1} & \cdots & R_{6,5}(q) \tilde{r}_{5} \tilde{l}_{6}^{2}(t ; v)
\end{array}\right]} \\
& v_{\text {tip }}(t ; v)=\left[\dot{l}_{1}^{1}(t ; v)^{\mathrm{T}} \dot{l}_{1}^{2}(t ; v)^{\mathrm{T}} \cdots \dot{l}_{6}^{1}(t ; v)^{\mathrm{T}} \dot{l}_{6}^{2}(t ; v)^{\mathrm{T}}\right]^{\mathrm{T}} \\
& \Lambda_{\text {tip }}(t)=\sigma \operatorname{diag}\left[\varepsilon_{1}^{1}(t) \varepsilon_{1}^{1}(t) \varepsilon_{1}^{2}(t) \varepsilon_{1}^{2}(t)\right. \\
& \left.\cdots \varepsilon_{6}^{1}(t) \varepsilon_{6}^{1}(t) \varepsilon_{6}^{2}(t) \varepsilon_{6}^{2}(t)\right]
\end{aligned}
$$

$$
\varepsilon_{i}^{j}(t)= \begin{cases}1 & \text { if leg } j \text { of module } i \text { is in } \\ 0 & \text { otherwise }\end{cases}
$$$$
i=1, \ldots, 6, j=1,2
$$ 
the left and right legs are numbered legs 1 and 2, respectively, $l_{i}^{j}(i=1, \ldots, 6, j=1,2)$ is the distance vector from yaw joint $i-1$ to the tip of leg $j$ of module $i$, and $\sigma$ is the damping coefficient of the reaction force.

We can write $\hat{q}^{\mathrm{T}}=\left[v t+x_{0} y_{0} 0 \cdots 0\right]$ and $\dot{\hat{q}}^{\mathrm{T}}=$

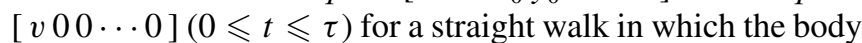
segments are aligned without producing body undulations for one stepping period, where $x_{0}$ and $y_{0}$ are, respectively, the values of $x$ and $y$ at $t=0$ and $\tau$ is the stepping period. We linearize the equation of motion (2) around the state of the straight walk by substituting $q=\hat{q}+\delta q$ into (2) and obtaining the first-order Taylor series expansion with respect to $\delta q$ to obtain $K(\hat{q}) \delta \ddot{q}$

$$
\begin{aligned}
= & -\left\{D+H^{\mathrm{T}} L_{\text {tip }}(\hat{q}, t ; v)^{\mathrm{T}} \Lambda_{\text {tip }}(t) L_{\text {tip }}(\hat{q}, t ; v) H\right\} \delta \dot{q} \\
& -\left\{P+H^{\mathrm{T}} L_{\text {tip }}(\hat{q}, t ; v)^{\mathrm{T}} \Lambda_{\text {tip }}(t) \dot{L}_{\text {tip }}(\hat{q}, \dot{\hat{q}}, t ; v) H\right\} \delta q .
\end{aligned}
$$

For $\delta z^{\mathrm{T}}=\left[\delta \dot{q}^{\mathrm{T}} \delta q^{\mathrm{T}}\right]$, the linearized equation is given by

$$
\delta \dot{z}=A(t ; v) \delta z
$$

where

$$
\begin{aligned}
& A(t ; v) \\
& \quad=\left[\begin{array}{cc}
A_{11}(t ; v) & A_{12}(t ; v) \\
I_{8} & O_{8 \times 8}
\end{array}\right] \\
& A_{11}(t ; v) \\
& \quad=-K(\hat{q})^{-1}\left\{D+H^{\mathrm{T}} L_{\mathrm{tip}}(\hat{q}, t ; v)^{\mathrm{T}} \Lambda_{\mathrm{tip}}(t) L_{\mathrm{tip}}(\hat{q}, t ; v) H\right\} \\
& A_{12}(t ; v) \\
& \quad=-K(\hat{q})^{-1}\left\{P+H^{\mathrm{T}} L_{\mathrm{tip}}(\hat{q}, t ; v)^{\mathrm{T}} \Lambda_{\mathrm{tip}}(t) \dot{L}_{\mathrm{tip}}(\hat{q}, \dot{\hat{q}}, t ; v) H\right\} .
\end{aligned}
$$

Since $l_{i}^{j}(t ; v)$ is periodic with the step cycle period $\tau$ and $i_{i}^{j}(t ; v)$ is constant, we obtain $A(t+\tau ; v)=A(t ; v)$.

\section{APPENDIX B: FURTHER ANALYSIS USING NONDIMENSIONAL PARAMETERS}

To further investigate the dynamical characteristics that produce a Hopf bifurcation, we analyze the equation using nondimensional parameters. Since each module has identical physical parameters, we put $m, j, l$, and $w$ for the mass, inertia, length, and width, respectively, for the modules in the simple model. We introduce a reference time $\xi$ and put $t=\xi \phi^{*}$ $\left(0 \leqslant \phi^{*} \leqslant \tau / \xi\right)$. We divide the translational and rotational equations of the linearization equation (A6) by $m l$ and $m l^{2}$, respectively, and then obtain

$$
\begin{aligned}
K^{*} \delta q^{* \prime \prime} & \\
= & -\left\{D^{*}+H^{\mathrm{T}} L_{\text {tip }}^{*}\left(\phi^{*} ; v^{*}\right)^{\mathrm{T}} \Lambda_{\text {tip }}^{*}\left(\phi^{*}\right) L_{\text {tip }}^{*}\left(\phi^{*} ; v^{*}\right) H\right\} \delta q^{* \prime} \\
& -\left\{P^{*}+H^{\mathrm{T}} L_{\text {tip }}^{*}\left(\phi^{*} ; v^{*}\right)^{\mathrm{T}} \Lambda_{\text {tip }}^{*}\left(\phi^{*}\right) L_{\text {tip }}^{\prime *}\left(\phi^{*} ; v^{*}\right) H\right\} \delta q^{*},
\end{aligned}
$$

where ()$^{\prime}$ indicates the derivative with respect to the nondimensional time $\phi^{*}$,

$$
\begin{aligned}
& \delta q^{*}=\left[\delta x / l \delta y / l \delta \theta_{0} \cdots \delta \theta_{5}\right]^{\mathrm{T}} \\
& K^{*}=H^{\mathrm{T}}\left(L^{* \mathrm{~T}} L^{*}+J^{*}\right) H \\
& L^{*}=\left[\begin{array}{c|cccc}
I_{2} & \tilde{\rho}^{*} & & & \\
I_{2} & \tilde{r}^{*} & \tilde{\rho}^{*} & & \\
\vdots & \vdots & \ddots & \ddots & \\
I_{2} & \tilde{r}^{*} & \ldots & \tilde{r}^{*} & \tilde{\rho}^{*}
\end{array}\right] \\
& J^{*}=\operatorname{diag}\left[00 j /\left(m l^{2}\right) j /\left(m l^{2}\right) \cdots j /\left(m l^{2}\right)\right] \\
& P^{*}=\operatorname{diag}\left[\begin{array}{llll}
0 & 0 & 0 & k \xi^{2} /\left(m l^{2}\right) \cdots k \xi^{2} /\left(m l^{2}\right)
\end{array}\right] \\
& D^{*}=\operatorname{diag}\left[\begin{array}{llll}
0 & 0 & 0 & d \xi /\left(m l^{2}\right) \cdots d \xi /\left(m l^{2}\right)
\end{array}\right]
\end{aligned}
$$

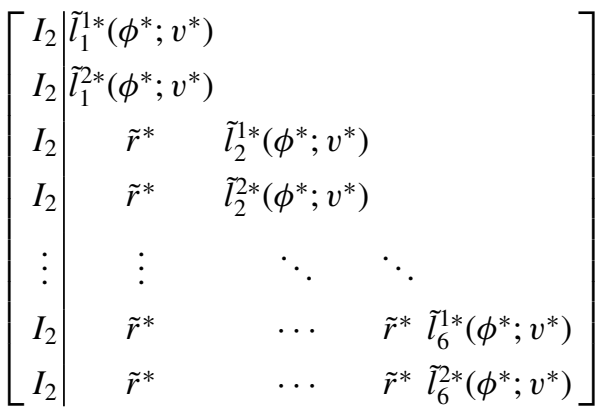$$
L_{\text {tip }}^{*}\left(\phi^{*} ; v^{*}\right)=
$$

$$
L_{\text {tip }}^{\prime *}\left(\phi^{*} ; v^{*}\right)=
$$

$$
\left[\begin{array}{c|ccc}
O_{2 \times 2} & \tilde{l}_{1}^{1 *}\left(\phi^{*} ; v^{*}\right) & & \\
O_{2 \times 2} & \tilde{l}_{1}^{2 / *}\left(\phi^{*} ; v^{*}\right) & & \\
O_{2 \times 2} & o_{2} & \tilde{l}_{2}^{1 *}\left(\phi^{*} ; v^{*}\right) & \\
O_{2 \times 2} & o_{2} & \tilde{l}_{2}^{2 *}\left(\phi^{*} ; v^{*}\right) & \\
\vdots & \vdots & \ddots & \ddots \\
O_{2 \times 2} & o_{2} & \ldots & o_{2} \tilde{l}_{6}^{1 / *}\left(\phi^{*} ; v^{*}\right) \\
O_{2 \times 2} & o_{2} & \ldots & o_{2} \tilde{l}_{6}^{2 / *}\left(\phi^{*} ; v^{*}\right)
\end{array}\right]
$$

$$
\begin{array}{r}
\Lambda_{\text {tip }}^{*}(t)=\sigma \xi / m \operatorname{diag}\left[\varepsilon_{1}^{1}\left(\phi^{*}\right) \varepsilon_{1}^{1}\left(\phi^{*}\right) \varepsilon_{1}^{2}\left(\phi^{*}\right) \varepsilon_{1}^{2}\left(\phi^{*}\right)\right. \\
\left.\cdots \varepsilon_{6}^{1}\left(\phi^{*}\right) \varepsilon_{6}^{1}\left(\phi^{*}\right) \varepsilon_{6}^{2}\left(\phi^{*}\right) \varepsilon_{6}^{2}\left(\phi^{*}\right)\right]
\end{array}
$$

$r^{*}=\left[\begin{array}{ll}0 & -1\end{array}\right]^{\mathrm{T}}, \quad \rho^{*}=\left[\begin{array}{ll}0 & -1 / 2\end{array}\right]^{\mathrm{T}}, \quad l_{i}^{j *}\left(\phi^{*} ; v^{*}\right)=$ $\left[p_{i}^{j}\left(\phi^{*} ; v^{*}\right) / l-(-1)^{j} w / l\right]^{\mathrm{T}}, \quad l_{i}^{j / *}\left(\phi^{*} ; v^{*}\right)=\left[\begin{array}{ll}-v^{*} & 0\end{array}\right]^{\mathrm{T}}$ $(i=1, \ldots, 6, j=1,2), v^{*}=v \xi / l$, and $p_{i}^{j}\left(\phi^{*} ; v^{*}\right)$ indicates the position of the tip of leg $j$ of module $i$ between the AEP and the PEP. This equation is governed by seven nondimensional parameters: $w / l, j /\left(m l^{2}\right), k \xi^{2} /\left(m l^{2}\right), d \xi /\left(m l^{2}\right), \xi / \tau, \sigma \xi / m$, and $v \xi / l$
[1] B. D. Anderson, J. W. Shultz, and B. C. Jayne, J. Exp. Biol. 198 1185 (1995)

[2] S. M. Manton, J. Linn. Soc. (Zool.) 46, 251 (1965).

[3] S. M. Manton, The Arthropoda: Habits, Functional Morphology and Evolution (Clarendon Press, Oxford, 1977).
[4] J. Gray, Animal Locomotion (Weidenfield \& Nicholson, London, 1968).

[5] R. M. Alexander and G. Goldspink, Mechanics and Energetics of Animal Locomotion (John Wiley \& Sons, New York, 1977). 
[6] R. D. Barnes, Invertebrate Zoology (The Dryden Press, Orlando, 1987).

[7] S. A. Wainwright, W. D. Biggs, J. D. Currey, and J. M. Gosline, Mechanical Design in Organisms (Princeton University Press, Princeton, 1982).

[8] A. H. Cohen, S. Rossignol, and S. Grillner, Neural Control of Rhythmic Movements in Vertebrates (John Wiley \& Sons, New York, 1988).

[9] A. H. Cohen, Brain, Behav. Evol. 40, 112 (1992).

[10] S. Grillner, Nat. Rev. Neurosci. 4, 573 (2003).

[11] S. Mori, Prog. Neurobiol. 28, 161 (1987).

[12] G. N. Orlovsky, T. Deliagina, and S. Grillner, Neuronal Control of Locomotion: From Mollusc to Man (Oxford University Press, Oxford, 1999).

[13] M. L. Shik and G. N. Orlovsky, Physiol. Rev. 56, 465 (1976).

[14] M. Golubitsky, I. Stewart, P. L. Buono, and J. J. Collins, Physica D 115, 56 (1998).

[15] M. Golubitsky, I. Stewart, P. L. Buono, and J. J. Collins, Nature 401, 693 (1999).

[16] R. M. Alexander, Philos. Trans. R. Soc. London B 338, 189 (1992).

[17] S. Aoi, T. Yamashita, and K. Tsuchiya, Phys. Rev. E 83, 061909 (2011).

[18] H. Geyer, A. Seyfarth, and R. Blickhan, Proc. R. Soc. B 273, 2861 (2006).

[19] P. Holmes, R. J. Full, D. E. Koditschek, and J. Guckenheimer, SIAM Rev. 48, 207 (2006).

[20] A. J. Ijspeert, A. Crespi, D. Ryczko, and J. M. Cabelguen, Science 315, 1416 (2007).

[21] K. Ohgane and K.-I. Ueda, Phys. Rev. E 77, 051915 (2008).

[22] K. Ohgane and K.-I. Ueda, Phys. Rev. E 81, 041909 (2010).

[23] M. Srinivasan and A. Ruina, Nature 439, 72 (2006).

[24] E. D. Tytell, C.-Y. Hsu, T. L. Williams, A. H. Cohen, and L. J. Fauci, Proc. Nat. Acad. Sci. USA 10719832 (2010).

[25] S. Aoi, H. Sasaki, and K. Tsuchiya, SIAM J. Appl. Dyn. Syst. 6, 348 (2007).

[26] S. H. Collins, A. L. Ruina, R. Tedrake, and M. Wisse, Science 307, 1082 (2005).

[27] J. Dean, T. Kindermann, J. Schmitz, M. Schumm, and H. Cruse, Auton. Robots 7, 271 (1999).
[28] H. Cruse, T. Kindermann, M. Schumm, J. Dean, and J. Schmitz, Neural Netw. 11, 1435 (1998).

[29] M. H. Dickinson, F. Lehmann, and S. P. Sane, Science 284, 1954 (1999).

[30] A. Jusufi, D. I. Goldman, S. Revzen, and R. J. Full, Proc. Natl. Acad. Sci. USA 105, 4215 (2008).

[31] H. Kimura, Y. Fukuoka, and A. H. Cohen, Int. J. Robot. Res. 26, 475 (2007).

[32] R. Pfeifer, M. Lungarella, and F. Iida, Science 318, 1088 (2007).

[33] R. E. Ritzmann, R. D. Quinn, and M. S. Fischer, Arthropod Struct. Dev. 33, 361 (2004).

[34] S. Steingrube, M. Timme, F. Wörgötter, and P. Manoonpong, Nat. Phys. 6, 224 (2010).

[35] B. Webb, Nature 417, 359 (2002).

[36] See Supplemental Material at http://link.aps.org/supplemental/ 10.1103/PhysRevE.87.012717 for the movies of the appearance of body undulations during robot locomotion; the effects of change in spring constants of torsional springs at yaw joints based on computer simulation.

[37] J. Guckenheimer and P. Holmes, Nonlinear Oscillations, Dynamical Systems, and Bifurcations of Vector Fields (SpringerVerlag, Berlin, 2002).

[38] S. H. Strogatz, Nonlinear Dynamics and Chaos: With Applications to Physics, Biology, Chemistry, and Engineering (Perseus Books, New York, 1994).

[39] T. R. Beal, AIAA J. 3, 486 (1965).

[40] M. A. Langthjem and Y. Sugiyama, J. Sound Vib. 238, 809 (2000).

[41] Y. P. Park and C. D. Mote, J. Sound Vib. 98, 247 (1985).

[42] J. J. Wu, J. Sound Vib. 42, 45 (1975).

[43] D. A. Crolla and F. D. Hales, J. Terramech. 16, 1 (1979).

[44] S. Tousi, A. K. Bajaj, and W. Soedel, Veh. Syst. Dyn. 21, 333 (1992).

[45] H. Troger and K. Zeman, Veh. Syst. Dyn. 13, 161 (1984).

[46] A. Minelli, D. Foddai, L. A. Pereira, and J. G. E. Lewis, J. Zool. Syst. Evol. Res. 38, 103 (2000).

[47] C. Guschlbauer, H. Scharstein, and A. Büschges, J. Exp. Biol. 210, 1092 (2007).

[48] J. Schmitz, J. Exp. Biol. 183, 15 (1993). 